\title{
Design of elliptically-vibrating ultrasonic actuator for nanocoining
}

Thomas A. Dow ${ }^{1,2}$. John Nowak ${ }^{1,2}$ and Jonas R. Kessing ${ }^{1,2}$

${ }^{1}$ Department of Mechanical and Aerospace Engineering, North Carolina State University, Raleigh, NC 27695, USA

${ }^{2}$ Precision Engineering Center Box 7918, Raleigh, NC 27695, USA

Email: dow@ncsu.edu

Corresponding Author:

Thomas A. Dow

dow@ncsu.edu

Box 7918

Raleigh, NC 27695

(919)-513-2172

Keywords: Nanocoining, ultrasonic transducer, nanoindentation, structured surfaces, diamond die, bio-inspired technology 


\section{Contents}

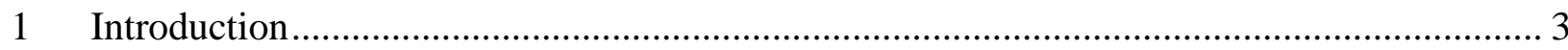

2 Process Requirements ………………………………............................................... 5

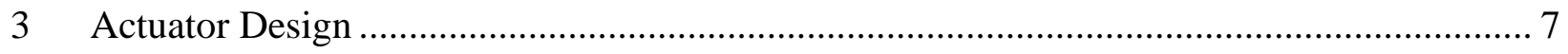

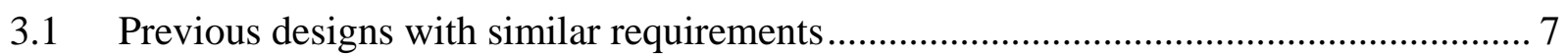

3.1.1 Elliptical Vibration Assisted Machining (EVAM) ……...................................... 7

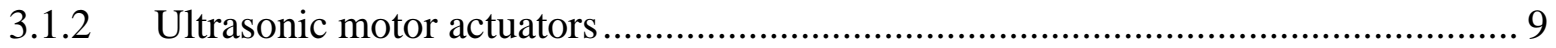

3.2 Performance requirements for the Nanocoining actuator ........................................... 11

3.3 Design Methodology ………………………….................................................. 13

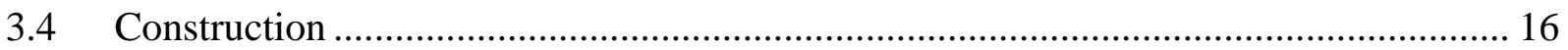

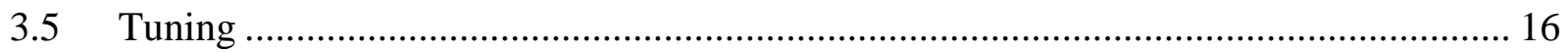

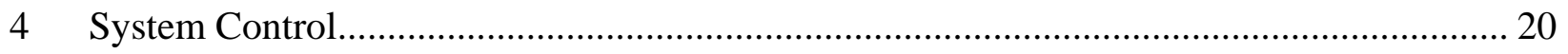

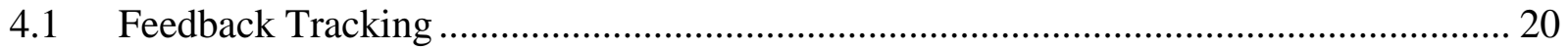

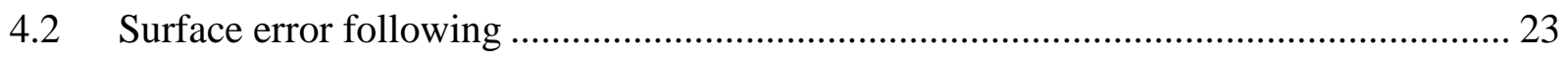

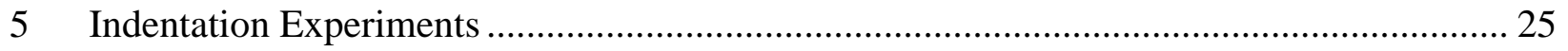

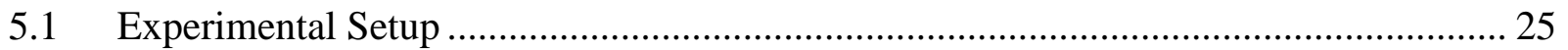

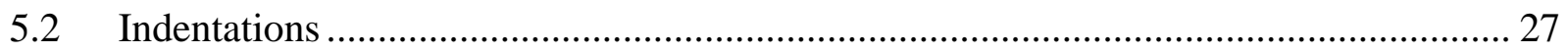

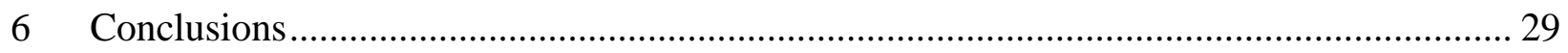

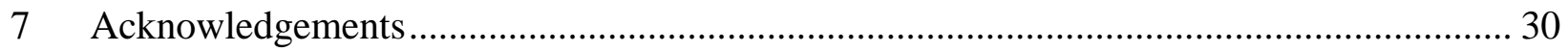

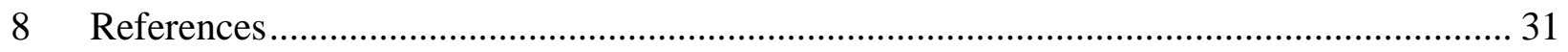




\begin{abstract}
Nanocoining is a method of rapidly creating a cylindrical mold surface covered with features smaller than the wavelength of light. This mold can then be used in a roll-to-roll process to make surfaces whose functionality depends on the wavelength of the illumination. The die replaces the typical diamond tool used to produce overlapping grooves for applications such as reflective signs. The die has a face area approximately $20 \mu \mathrm{m}$ square that has been patterned in an FIB. It is mounted on a 2D ultrasonic actuator and follows an elliptical path that matches the surface speed of the moving workpiece during the short contact time and creates approximately 6000 features per impact. The spacing of die indents are controlled by the speed of the Diamond Turning Machine axes such that a small overlap exists from previous indents as the die spirals around and along the mold surface. Because the die is small, the indentations must occur rapidly to make nanocoining a feasible process. This work focuses on the design and control of a nominally $40 \mathrm{kHz}, 2 \mathrm{D}$ resonant actuator that is suitable for this process. A controller to automatically track resonance is described to maintain the elliptical motion during indentation. Methods of tuning the behavior of the actuator and maintaining a constant indent depth are proposed. Finally, $500 \mathrm{~nm}$ pitch feature indents were created on a brass workpiece at $40 \mathrm{kHz}$ and scanning electron microscope (SEM) images of the features are provided.
\end{abstract}

\title{
1 Introduction
}

Bio-inspired structured surfaces are commonly used in developing coatings that can exhibit a variety of traits, including anti-reflective (AR), superhydrophobic or superhydrophilic qualities. AR surfaces are of particular interest due to their applications in optical lenses, solar cells, and photosensitive detectors [1]. To achieve anti-reflective properties, these surfaces are designed to mimic the nanopillar structures found on the surfaces of moth and butterfly eyes, or 
cicada and hawkmoth wings. In nature, these AR properties offer survival advantages including camouflage from predators and increased visibility in low light settings [2, 3]. Smaller than the wave length of light, these periodic features introduce a graded index of refection that reduces the amount of reflection compared to an unstructured surface [4]. Moth eye inspired structures have demonstrated an order of magnitude less reflectance over traditional AR thin films in the visible light and near-infrared spectrum [5].

Some of the existing technologies used to generate these nanostructures include interference lithography, etching, and bio-templates [6-8]. Linear gratings can be generated using interference lithography, where two interfering argon ion laser sources are used to create a sinusoidal intensity pattern to which photoresist is exposed [6]. The photoresist surface can then be rotated and exposed to the sources again, producing an array of nanofeatures. Nickel replicates of the photoresist structures were produced and used in an embossing process on acrylic and polycarbonate glass. Another technique for producing nanostructures involves using a bio-template, whereby an anti-reflective biological sample such as the eye of moth or the wing of a cicada is used as a mold to replicate the desired features. In [7], a cicada's wing is replicated by first thermally depositing a thin gold layer on the specimen resulting in a mold of the negative of the features. This gold mold was then deposited with PMMA polymer film to produce a replica of the circada wing features. Non-lithographic etching techniques were used to produce moth eye structures, where monolayer silica colloidal crystals are used as etching masks on the surface of the silicon wafer [8]. Once an array of silica particles is positioned on the wafer, reactive-ion etching (RIE) is used to remove the silicon exposed around the particle mask. After the RIE is complete, the silica particles are removed using a hydrofluoric acid wash, revealing the array of silicon nanopillars resembling moth eye structures. While existing techniques are 
successful in replicating these bio-inspired patterns, they often are long in duration and require several manufacturing steps to complete (some steps in the lithographic process can take up to 11 hours [9]).

Nanocoining is a mechanical approach to rapidly create surface structures using a small diamond die $(20 \times 20 \mu \mathrm{m})$ containing an array of 6400 nanofeatures machined with a focused ion beam (FIB). This die is pressed into a diamond-turned surface, leaving a field of sub-micrometer features. To create a continuous field of the desired features, the indents are tiled together on the surface of the workpiece. Due to the small size of the die, the indents must quickly cover relatively large areas with nanofeatures in small amounts of time. Previous work at the Precision Engineering Center has shown high-quality nanocoined surfaces at $1 \mathrm{kHz}$ [10] using a nonresonant actuator, however indenting must occur at a much higher frequency for this process to be industrially relevant. To achieve high-frequency indenting, a resonant actuator design must be created with a control system that will maintain resonant actuation with the desired amplitude.

In this paper, the details relevant to the design and optimization of an actuator for nanocoining will be described. The actuator design will be explained by first discussing the performance requirements followed by the design methodology, construction features and tuning techniques. The controllers used to maintain resonance as well as a secondary actuator to track the work surface topology will be described. Finally, results of the system performance will be validated with nanocoining experiments.

\section{Process Requirements}

To create a continuous area of features, the individual die indents must be tiled together. One way this is done is by indenting the surface of a rotating drum on a DTM as shown in Figure 
1(a). The die must be held by an actuator that pushes it into the surface to replicate the die features while moving tangentially at a speed that matches the surface of the drum during contact to avoid smearing of the individual features. The drum surface speed is controlled by the spindle motor rpm and the radius of the drum. In addition to matching the drum speed during impact, the die must land one die width ahead of its position on the last cycle. This defines an "upfeed" distance that sets the relationship between the frequency of the die motion, its size and the surface speed of the drum. The "crossfeed" motion of the linear axis holding the die must advance along the drum at one die-width per revolution.

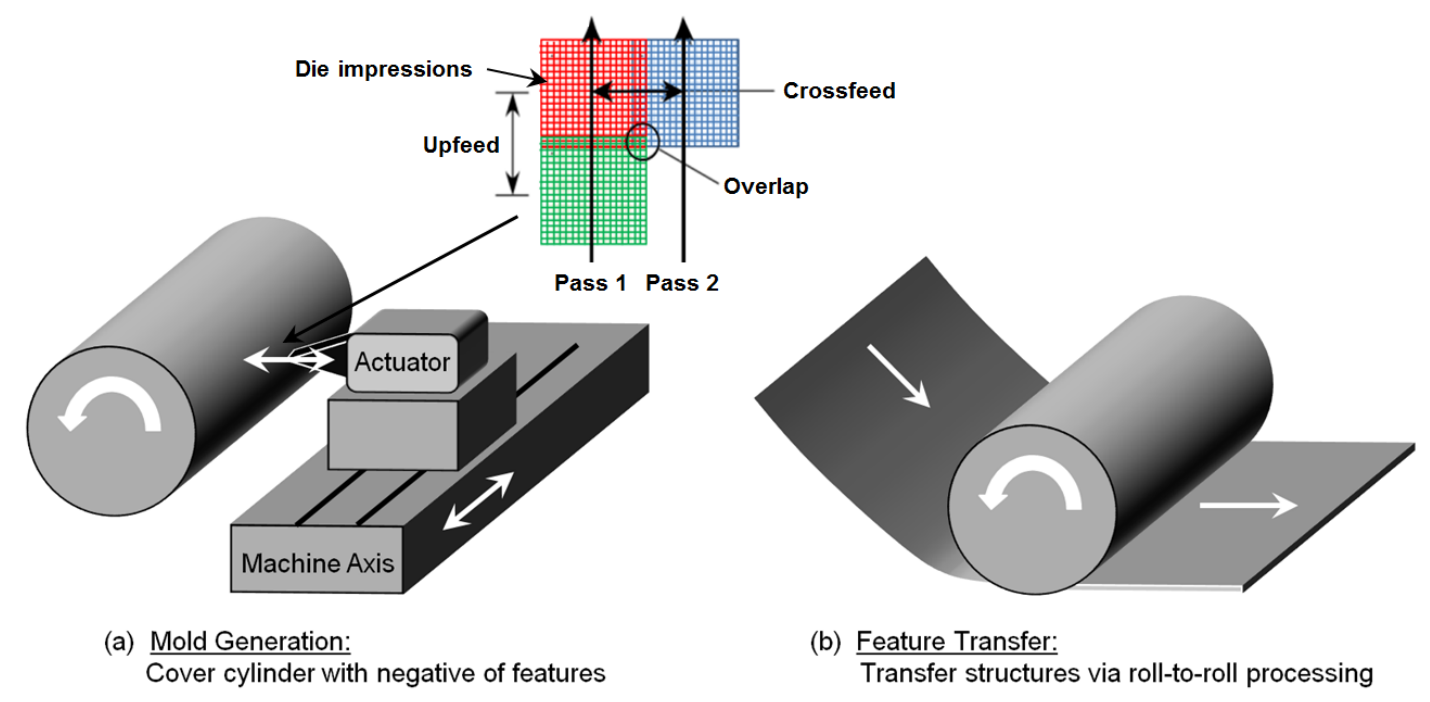

Figure 1. (a) Nanocoining of subwavelength surface features on a cylindrical mold using the ultrasonically vibrating diamond die and (b) roll-to-roll replication using the indented mold .

The registration problem is lessened due to the independent requirements for upfeed and crossfeed of the die. There is no requirement that the dies line up on each rotation of the drum only that the surface is fully covered by features. In fact, small overlap between successive die indents is desirable to avoid regions without features which would produce visible diffraction at a frequency related to size of the die. Once the drum is uniformly covered with the desired 
structures, it is used as a mold in a roll-to-roll process, for example, to rapidly generate features on a flexible plastic medium (Figure 1(b)).

\section{Actuator Design}

\subsection{Previous designs with similar requirements}

The requirements for the die actuator are described above and the larger the die and the higher its operating frequency, the shorter time it takes to cover the mold with features. The development of the actuator is based on research at the PEC and elsewhere to create high-speed motion with a path that will replicate, with high fidelity, the die features onto the mold surface.

\subsubsection{Elliptical Vibration Assisted Machining (EVAM)}

The actuators used for Elliptical Vibration-Assisted Machining are similar to those visualized for Nanocoining. An often cited review paper by Dow [23] discussed the process and the actuators concepts developed beginning in 1995.

EVAM is a method of machining that adds a small amplitude, high frequency elliptical tool motion and creates intermittent contact between the tool and workpiece as shown in Figure 2. The goal was to change the chip/tool motion such that the tool would move faster in the vertical direction than the chip and the friction would lift the chip rather than retard it. With an elliptical path, the tool moves cyclically out of the workpiece along with the chip. Because the relative speed between the chip and tool is small, average frictional effects are reduced, which results in an increased shear angle and reduces the cutting force, reducing temperature and prolonging tool life [21,22]. 


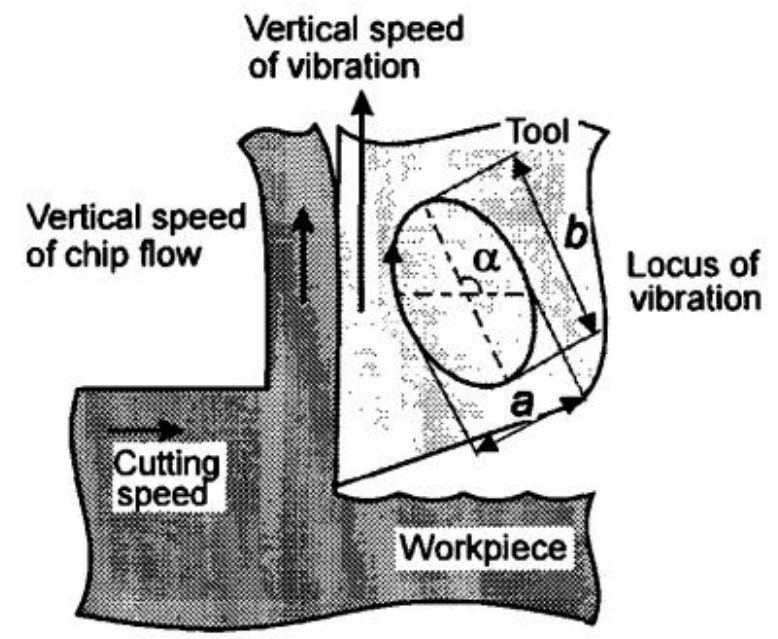

Figure 2. Schematic of EVAM process [22]

Early systems at the PEC used frequencies on the order of $1 \mathrm{kHz}$ to study the basic physics of the process. To increase the machining rate and avoid audible high frequency noise, EVAM tools have been designed to operate at ultrasonic frequencies (> $20 \mathrm{kHz})$. An example is shown in Figure 3 [21] of an elliptically-vibrating resonant actuator that operates at $20 \mathrm{kHz}$. The actuator body is symmetric about two bending mode directions creating a 2-D elliptical motion when excited at the resonant frequency. Two sets of opposing piezoelectric elements are excited with sine wave voltages out-of-phase to produce the elliptical path in the plane transverse to the length of the actuator. To avoid unwanted damping losses and maximize displacement, the structure is mounted at vibration nodes $[3,4]$. 


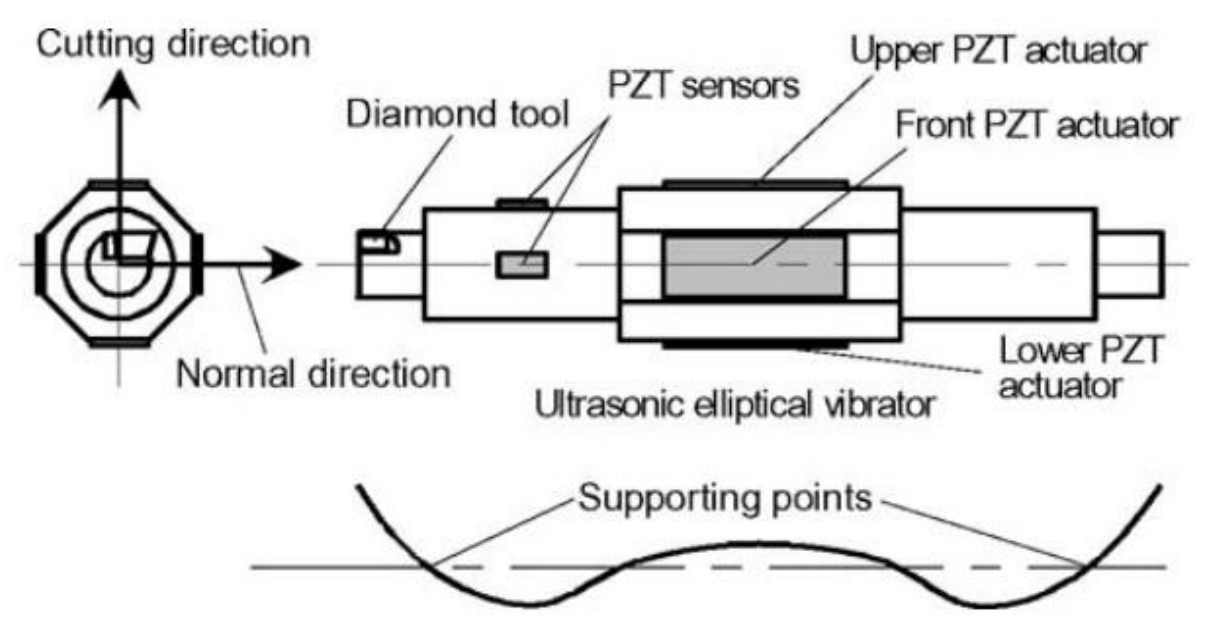

Third resonant mode of bending

Figure 3. Ultrasonic bending mode elliptical vibrator for EVAM [21]

\subsubsection{Ultrasonic motor actuators}

Another example of 2D ultra-sonic piezoelectric actuators developed at the PEC for linear or rotary motion is shown in Figure 4. This actuator utilizes longitudinal and bending modes of vibration of a piezoelectric material driven by a single high frequency signal to move the slide. The longitudinal vibration mode changes the load on the surface and the bending mode moves the tip in the direction of the slide motion. The tip of the device displaces about $1 \mu \mathrm{m}$ in an elliptical path at $50 \mathrm{kHz}$, which is one of the resonant frequencies of the structure. By changing the phase of the tangential and longitudinal vibrations, the shape of the path can be modified to change the driving direction or the speed of the tip.

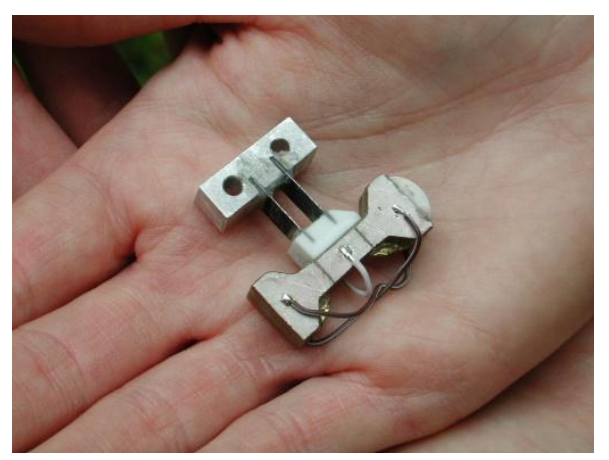

Figure 4. Photograph of the 2D ultrasonic motor for air bearing spindle 
This design indicates the potential of small piezoelectric designs to operate at high frequency. A photograph of the ultrasonic actuator is shown in Figure 5. For this drive application, the actuator was spring loaded against the slideway with a low normal force. When excited at the natural frequency, the normal force changed each cycle and the lateral vibration would have different frictional force depending on the normal force. As a result, the slideway would move to the left or right depending on the phase difference between the longitudinal and the bending mode.

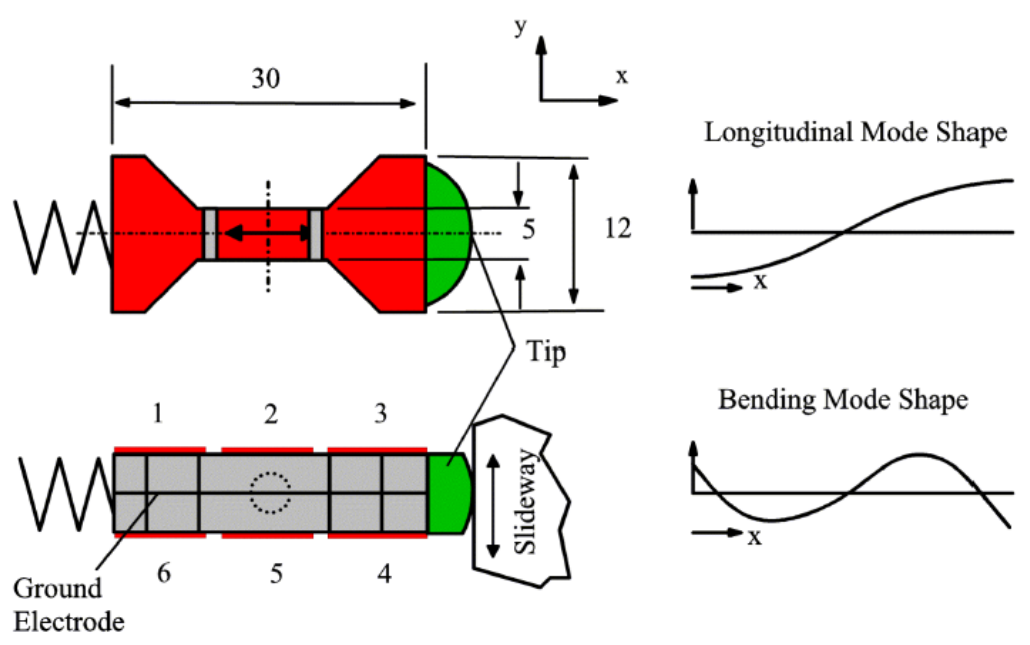

Figure 5. Ultrasonic piezoelectric actuator design. The numbers in the side view at left indicate the size of the actuator in $\mathrm{mm}$. This design has three active elements and the six electrodes are indicated in the top view at bottom left. The graphs in the middle are the mode shapes at $50 \mathrm{Khz}$ and the photograph shows the relative size of the actuator [24].

The actuator shown in Figure 5 operates at a resonant frequency well above the first mode. The key is to design the shape of the actuator (note the dog bone design) such that there are two modes of vibration at the same frequency and the motion of the tip at this frequency produces the desired path. While the actuator in Figure 5 worked well for the motor application, 
it is not appropriate for nanocoining because of the sliding that occurs at the interface. A more controlled motion is necessary to successfully coin sub-micrometer features.

\subsection{Performance requirements for the Nanocoining actuator}

Generating tiled arrays of indents on the mold at $40 \mathrm{kHz}$ requires the surface to move at a velocity such that it travels the length of the die $(20 \mu \mathrm{m})$ during every period of the actuator oscillation $(25 \mu \mathrm{sec})$. The work surface is moved a constant rate based on the actuator frequency and die size and the result will be a succession of indents precisely following the previously formed features. When indenting into a translating workpiece, the die must match the tangential velocity of the drum while the die is in contact with the surface. If this condition is not achieved, the shape of the die features will be distorted or smeared.

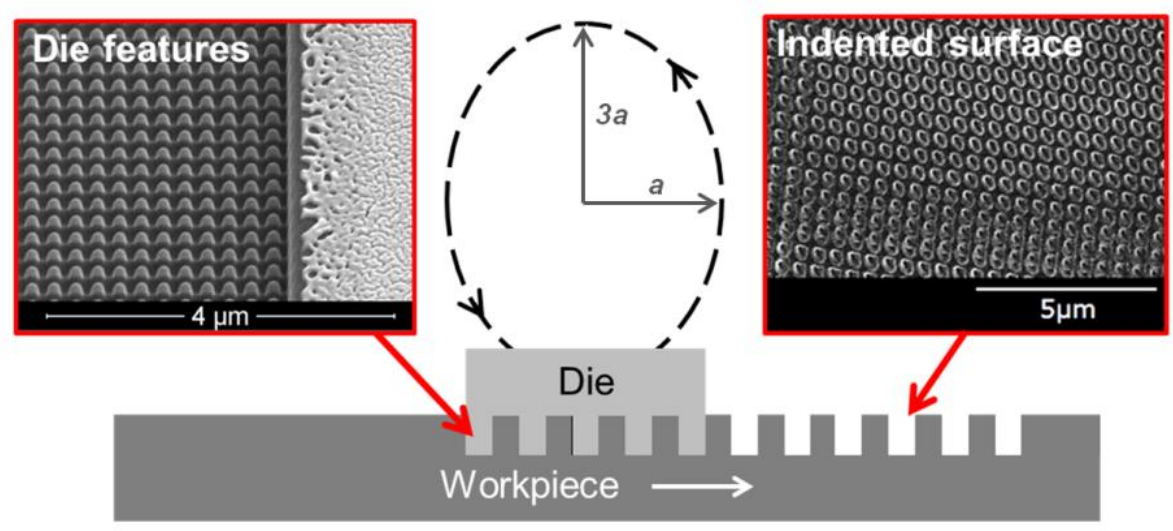

Figure 6. Vertical and horizontal dimensions of elliptical indenting path.

To minimize the distortion caused by a velocity mismatch, the die must be moved in a $2 \mathrm{D}$ path that allows the die to be pressed normal in to the surface while simultaneously matching the tangential speed of the workpiece. This goal can be achieved by moving the die in an elliptical path as shown in Figure 6 but only approximately. Since the workpiece is moving at a constant speed, the die actuator motion can be synchronized to match that speed at the bottom of the elliptical path in Figure 6. However, there has to be some small indentation distance so the die and workpiece will contact over some finite time. This means that an elliptical path cannot 
achieve a pure indentation motion. However, if the ellipse is taller than it is wide and the indentation distance is on the order of $200 \mathrm{~nm}$, the smearing of individual die features will be less than $20 \mathrm{~nm}$. While not perfect, the elliptical die motion is the only reasonable die path to achieve the two orthogonal motions using a single drive frequency.

Assuming the maximum horizontal speed of the die and the workpiece surface are equal during contact, the required dimension of $a$ is a function of the die size and can be expressed mathematically in the following form [10]:

$$
a=\frac{L_{\text {die }}}{2 \pi}
$$

where $L_{d i e}$ is the length of the die measured in the direction of motion of the mold. If the required dimension $a$ cannot be achieved with the actuator, a smaller indenting ellipse can still be used by slowing the speed of the workpiece. This, however, results in overlapping of successive indents and can increase the time needed to cover a given area. The spindle speed is set to match the measured dimension $a$ using the expression:

$$
\omega_{\text {spindle }}=\frac{60 f a_{\text {meas }}}{r_{w p}}
$$

where $\omega_{\text {spindle }}$ is the spindle speed in RPM, $f$ is the actuator frequency in $\mathrm{Hz}, a_{\text {meas }}$ is the horizontal dimension of the die path in $\mathrm{mm}$, and $r_{w p}$ is the radius of the cylindrical workpiece in $\mathrm{mm}$.

The vertical ellipse dimension dictates the time it takes for the die to enter and exit contact with the part, whereby increasing the vertical amplitude decreases the amount of indentation time between the die and workpiece. Based on the die size, the design goal was to produce a $3 \times 9 \mu \mathrm{m}$ elliptical path at $\sim 40 \mathrm{kHz}$. 


\subsection{Design Methodology}

The elliptical indenting motion is generated at $40 \mathrm{kHz}$ using a $2 \mathrm{D}$ beam-type resonant actuator. The actuator structure is designed such that two orthogonally vibrating modes occur at the same frequency so a single input frequency can excite both directions of motion. In Figure 7, the superimposed motion of the vibrating modes results in an elliptical motion, if they are excited out of phase. The front end of the actuator decreases in cross-sectional area to act as an ultrasonic concentrator that will amplify resonant displacement in ultrasonic applications $[11$, 12].

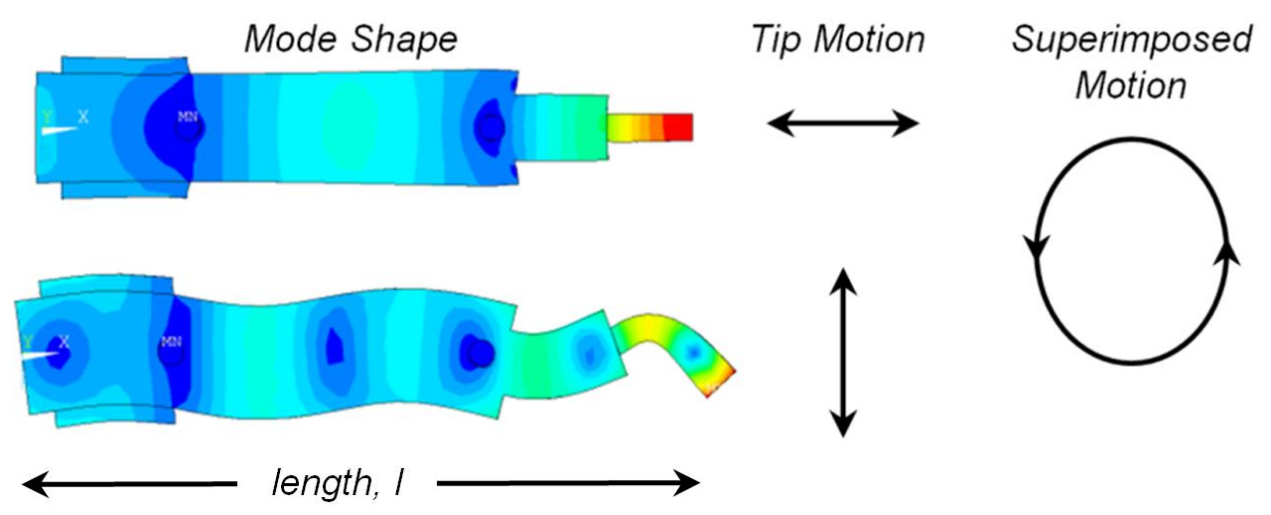

Figure 7. Longitudinal and transverse vibration modes generating elliptical motion.

Finite element analysis (FEA) was used to design the actuator shape to exhibit the desired resonant behavior. The design is based on uniform beam in a free-free orientation, where the longitudinal natural frequency $\omega_{n, L}$ and bending natural frequency $\omega_{n, B}$ can be expressed analytically with the following equations [13]:

$$
\begin{aligned}
& \omega_{n, L}=\frac{n \pi c}{l} \\
& \omega_{n, B}=(\beta l)^{2} \sqrt{\frac{E I}{\rho A l^{4}}}
\end{aligned}
$$

where 
$n=$ mode number

$c=$ wave speed of the beam material

$E=$ Young's Modulus

$I=$ area moment of inertia

$\rho=$ material density

$l=$ length of the beam

$A=$ cross-sectional area of the beam

$\beta l=$ function of the mode number and boundary conditions

The actuator dimensions were chosen to match the longitudinal and bending modes of interest. Considering a uniform aluminum beam with a width of $12.7 \mathrm{~mm}$ and a height of 10 $\mathrm{mm}$, the $2^{\text {nd }}$ longitudinal and the $5^{\text {th }}$ bending mode frequencies are plotted as a function of beam length in Figure 8. This graph shows there is a point where the two modes vibrate the same frequency and can be simultaneously excited.

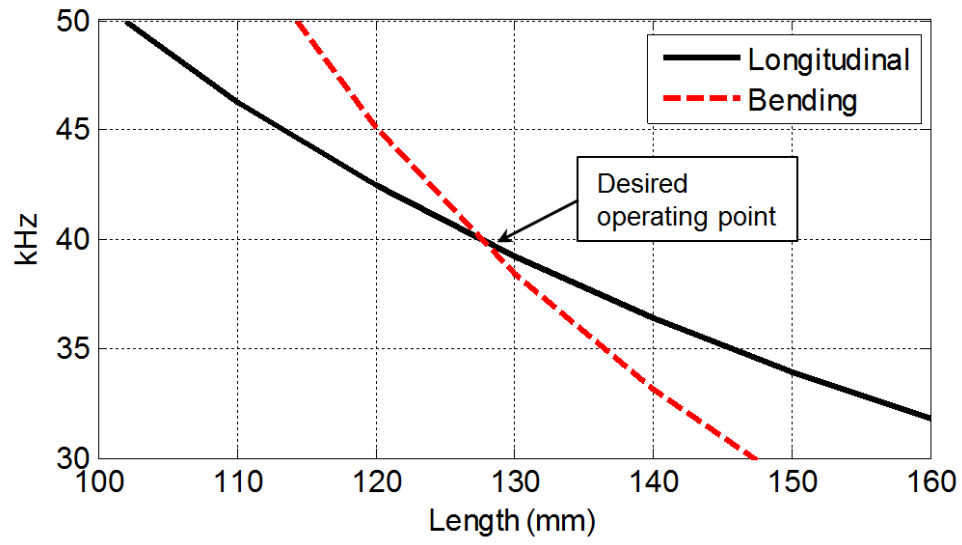

Figure 8. Theoretical longitudinal and transverse mode frequencies of a uniform aluminum beam of varying beam lengths. 
The goal of the actuator design process is to match the frequency of the longitudinal and bending mode for elliptical motion while maximizing the vibrational amplitude. The design steps for a 2D resonant actuator are as follows:

1. Calculate beam size: Use analytical solutions for uniform beam to estimate required dimensions based on desired operating frequency.

2. Select vibration modes: Use FEA to model the beam and ensure that the two desired operating modes share at least two displacement nodes for mounting. Resize using Step 1 if necessary.

3. Add piezoelectric actuators: Model piezoelectric elements to excite the beam in the desired directions. Since the deflection of the actuator is small compared to the beam ( $500 \mathrm{~nm}$ vs. $5 \mu \mathrm{m}$ ), it can restrict the vibration of the resonating beam. The actuators are mounted close to the vibration nodes where the beam deflection is smaller (compared to the tip) to minimize damping effects.

4. Reshape tool end: Reducing the cross-sectional area near the tool end of the actuator increases the vibrational amplitude.

5. Adjust height/length: Since material was added or removed (different actuator size and position and reshaping), the overall height and length of the actuator must be adjusted to maintain the desired resonant frequency. The sensitivities of the two modes to dimension changes can be deduced from Equation (3) where the longitudinal mode is represented as a function length, whereas the transverse mode is a function of length and cross-sectional area.

6. Verify node locations: Ensure the selected nodes for mounting are retained for both modes. Geometry modification/actuator placement can be used to realign nodes. 


\subsection{Construction}

The actuator is constructed from a single piece of CNC machined 6061 aluminum and two PKI802 piezoelectric elements. The piezos are fixed to the body of the actuator using electrically conductive epoxy, such that the exposed electrode of the each piezo is the signal voltage input and the epoxy side of the piezo and aluminum actuator body is electrical ground. Four oval tip set screws are threaded though the sidewall and hold the actuator at the displacement node positions. Clearance between the sidewalls and actuator is kept to minimum (less than $1 \mathrm{~mm}$ ) to maximize longitudinal stiffness of the mount. The prefabricated diamond die is aligned and fastened to the tip of the actuator using 3M Scotch-Weld DP 460 (Figure 9).

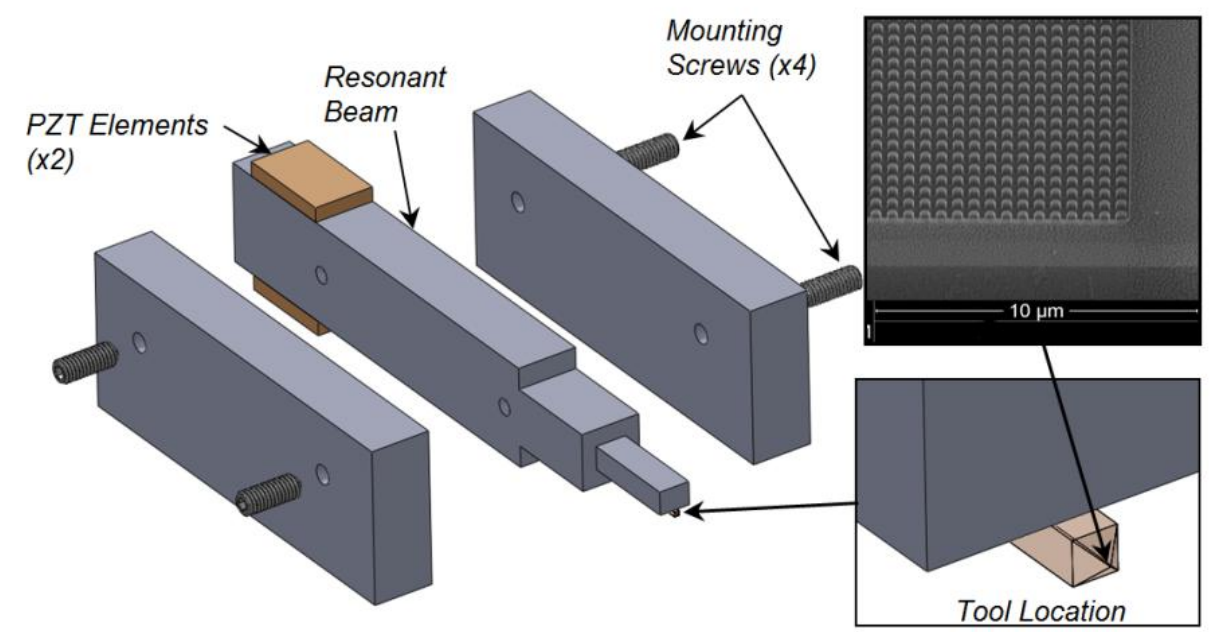

Figure 9. Assembly of actuator with die mounted at tip.

\subsection{Tuning}

While the goal is to match to orthogonal vibration modes, uncertainties such as dimensional errors in the construction process, behavior of the epoxy used to fix the piezo elements to the beam, node locations, and mount contact points affect the resonant frequencies and are not easily captured by the finite element model. Several methods have been developed for tuning the resonant frequencies such that each of the active vibration modes can be adjusted 
to occur at the same frequency despite model/fabrication discrepancies. When a difference in the two desired resonances is measured, a systematic method of moving the mode frequencies relative to each other is required. Methods such as selectively modifying the geometry of the actuator after assembly and strategically adding mass to the system can be used to tune the resonant frequencies. However, this can also change the location of the nodes which will result in a decrease in amplitude.

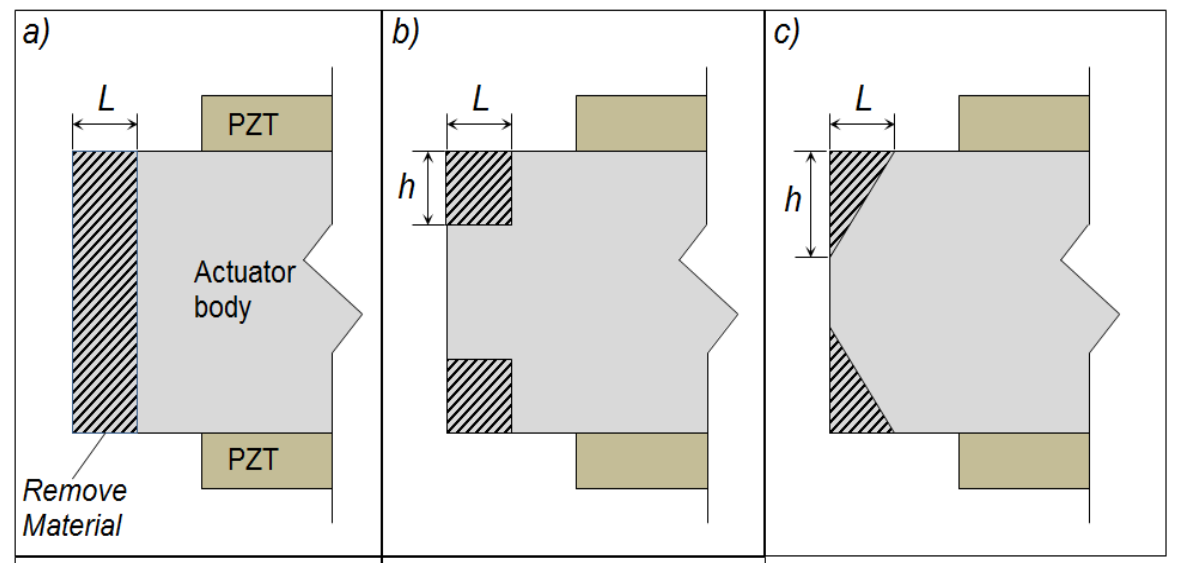

Figure 10. Methods of tuning the resonant frequencies of the actuator by removing material.

Figure $10(a-c)$ illustrates the methods used to tune the resonant response of the system These techniques involve removing material to change the effective length and mass distribution of the structure. It can be deduced from the analytical solution of a uniform beam natural frequency presented in Equation (3) that changing the length of the beam will alter the natural frequency of both the longitudinal and bending mode. Additionally changing the mass distribution while retaining the effective overall length (as shown in Figure 10(b) and (c)) should impact the bending mode differently than the longitudinal and ultimately result in the mode frequencies changing relative to each other. Modal and harmonic analyses were performed using 
the mechanical ANSYS FEA package to calculate the effect each geometry change would have on the actuator.
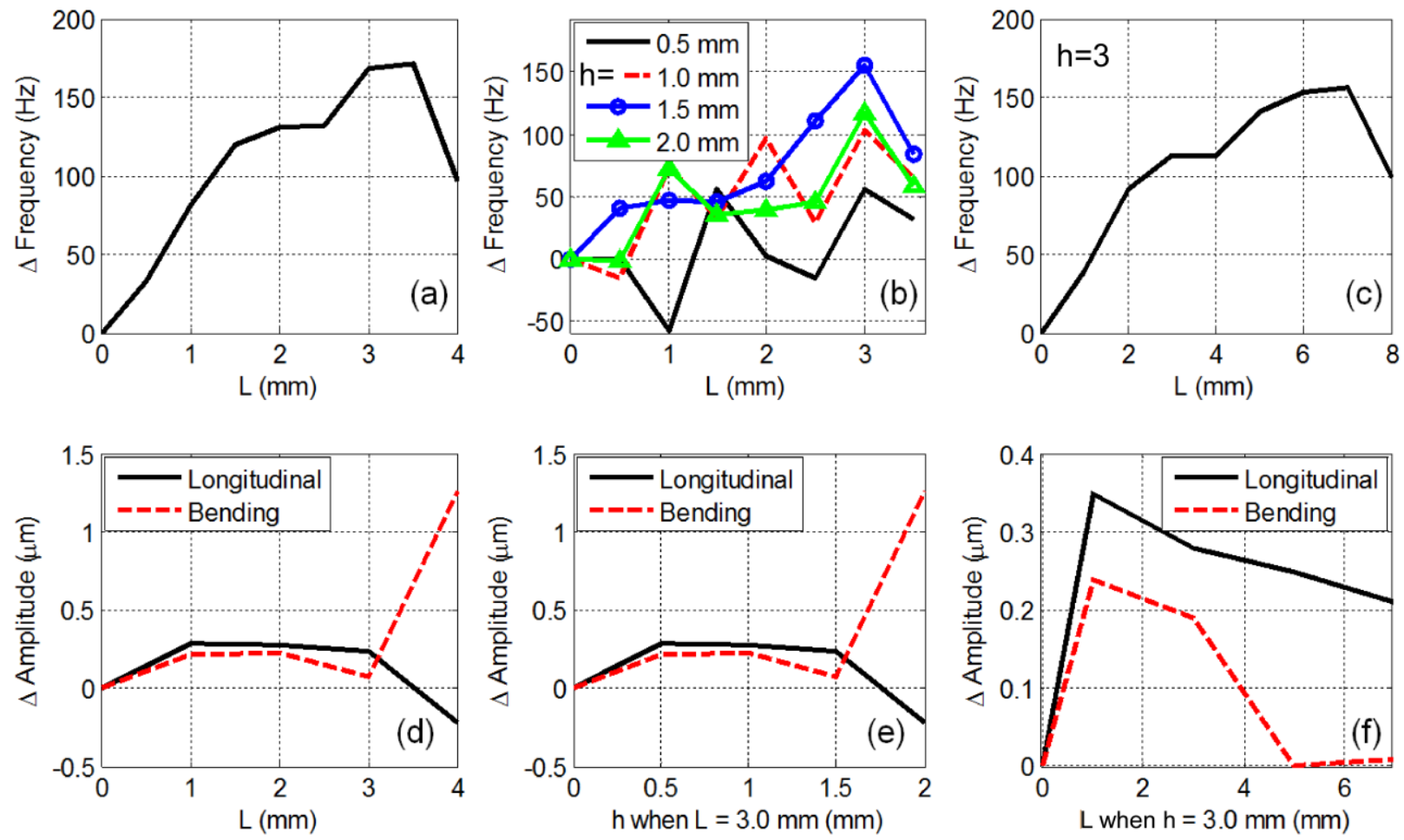

Figure 11. ANSYS results for frequency-tuning modifications where the bending mode is increased relative to the longitudinal mode.

Figure 11 illustrates the changes in frequency and amplitude as a result of the modifications to the structure shown in Figure 10. The plots in Figure 11 (a), (b) and (c) are the relative change in resonant frequency between the longitudinal and bending modes from the changes shown in Figure 10 (a), (b) and (c), respectively, where $\Delta$ Frequency is the difference between the longitudinal and bending mode frequency. Figure 11 (d), (e), and (f) are the change in output amplitude of the actuator for the longitudinal and bending mode. Modeling the amplitude response is important to ensure that the tuning modifications do not adversely interfere with the behavior of the actuator. It should be noted that the changes in geometry in Figure 10 
increase the bending mode relative the longitudinal mode, so for instances where the initial response shows the longitudinal mode lower than the bending mode another strategy is needed.

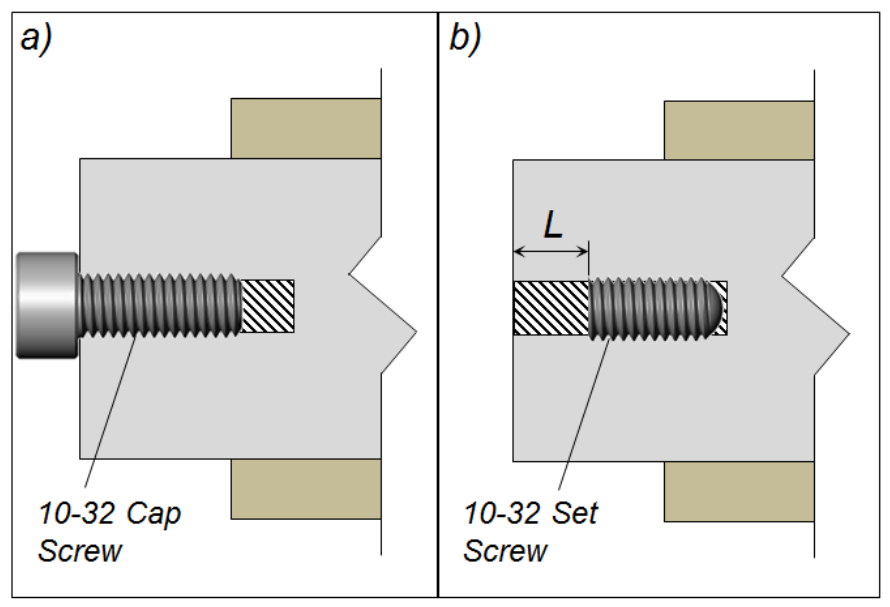

Figure 12. Tuning resonant frequency by adding mass using a cap screw (a) and a set screw (b).

To increase the longitudinal mode relative to the bending mode (the opposite effect of the strategies illustrated in Figure 10), a method of adding mass to the structure was introduced. Figure 12 shows the technique using a steel cap screw (a) or set screw (b) threaded into the rear of the actuator. These screw types allow different amounts of mass to be added to the system and the head of the cap screw adds a larger mass than the set screw. In addition, the dimension $L$ in Figure 12(b) allows adjustment of the center of mass which can fine tune the mass distribution of the actuator. The addition of this steel mass increases the frequency of the bending mode and corrects for measured resonant frequency differences. The method illustrated in Figure 12(b) is employed to correct the measured mismatch in resonant frequencies. Figure 13(a) and (b) are the measured response of the actuator before and after tuning, respectively. It is apparent that prior to tuning, the longitudinal and bending resonant peaks were $300 \mathrm{~Hz}$ apart, whereas the tuned actuator shows the resonant peaks occurring at nearly the same frequency. 

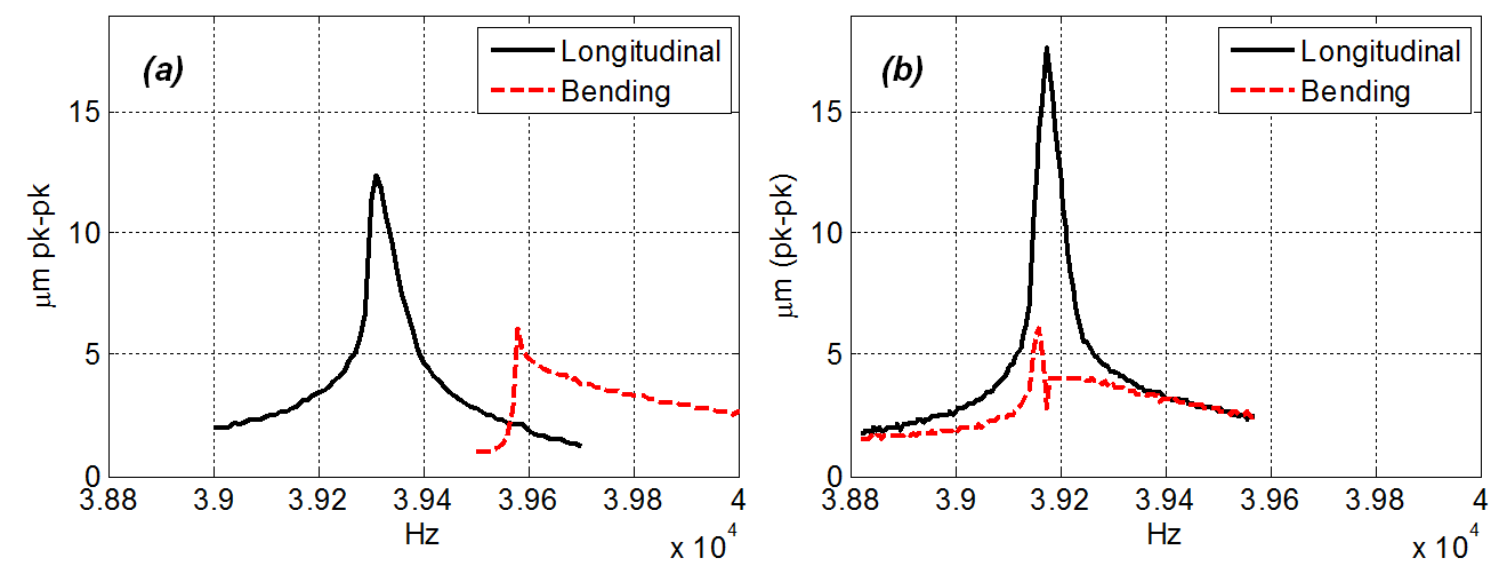

Figure 13. Frequency response of actuator before tuning (a) and after tuning (b). The amplitude of longitudinal vibration is about twice that of the bending motion at $39.15 \mathrm{kHz}$.

\section{$4 \quad$ System Control}

To generate consistent indents, a feedback controller is needed to maintain the elliptical motion of the vibrating actuator. A tracking mechanism to compensate for part and spindle errors in real time to maintain a constant depth of indentation was also constructed and tested.

\subsection{Feedback Tracking}

During extended operation, the resonant frequency of the system can drift as a result of a well-documented phenomenon, such as piezo self-heating effects, room temperature variations, and other environmental influences $[14,15]$. This change in resonance can be observed in the change in actuator amplitude for a constant-frequency input signal. As the resonance of the system drifts, the output amplitude typically decreases. A method for correcting the drift must be implemented to maintain a constant amplitude output. Failing to do so would results in uneven depths of the indents.

Automatic resonant frequency tracking is performed by measuring the phase between the current and voltage across the piezo inputs. Due to the interconnection of the piezoelectric actuators and aluminum beam, the electrical phase is a direct measurement of the mechanical 
response of the beam. This concept can be illustrated using classical 1-D modeling techniques for ultrasonic transducers, where the electromechanical system is modeled using a lumpedparameter, equivalent circuit model $[16,17]$. Figure 14 illustrates the frequency response of a simulated 1-D piezo-driven, ultrasonic transducer that demonstrates this characteristic relationship between the resonant response of the system and a current-to-voltage phase. The piezo acts as a self-sensing actuator, where the phase can be fed back to the controller and changes to the input frequency can be made to track a phase setpoint that corresponds to mechanical resonance. This concept is often referred to as minimum impedance tracking because mechanical resonance corresponds to minimum electrical impedance [18-20].

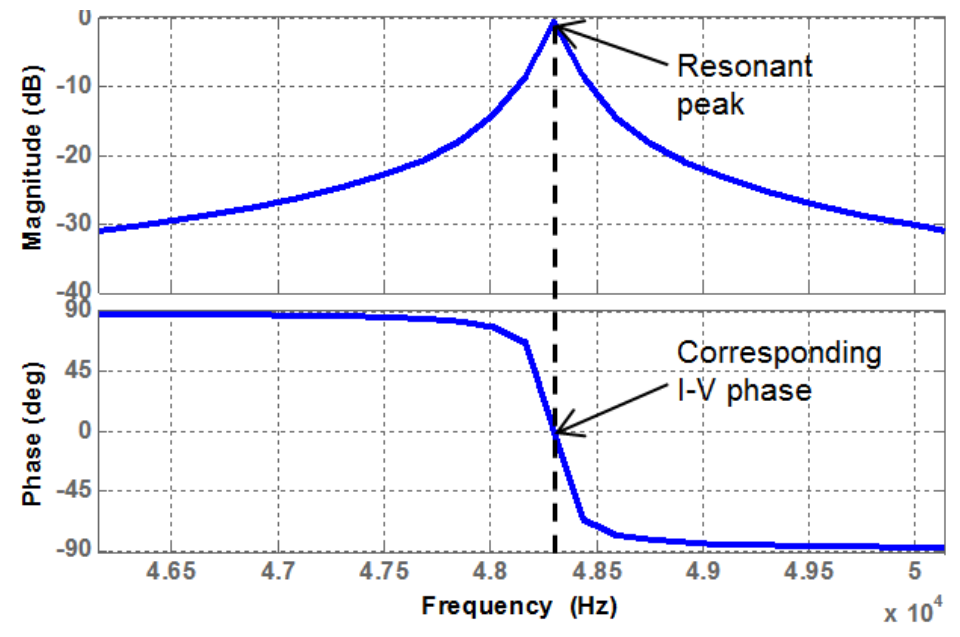

Figure 14. Ideal frequency response of a piezoelectric resonator.

The resonance tracking controller utilizes a lock-in amplifier in Figure 15 to internally compare the measured current signal across the piezo with the voltage drive signal it is generating. The lock-in amplifier synchronizes the current and voltage signal to a user-set phase difference (controlled to approximately $1^{\circ}$ ) by modulating the frequency of the output voltage. Since a constant phase angle corresponds to resonance, the resonant frequency of the actuator is automatically followed by the frequency the lock-in amplifier is generating. A signal 
conditioning circuit is used to split the single drive frequency and time-delay one channel, to produce two out-of-phase inputs for the actuator. A user interface for the controller was developed to program the phase between the two piezo elements $(\varphi)$ and amplitude $(A)$ of the input signals based on the desired motion of the actuator. Setting the input phase to a constant $90^{\circ}$ will excited both the longitudinal and bending modes, whereas a $0^{\circ}$ or $180^{\circ}$ phase will excited only the longitudinal or bending modes, respectively.

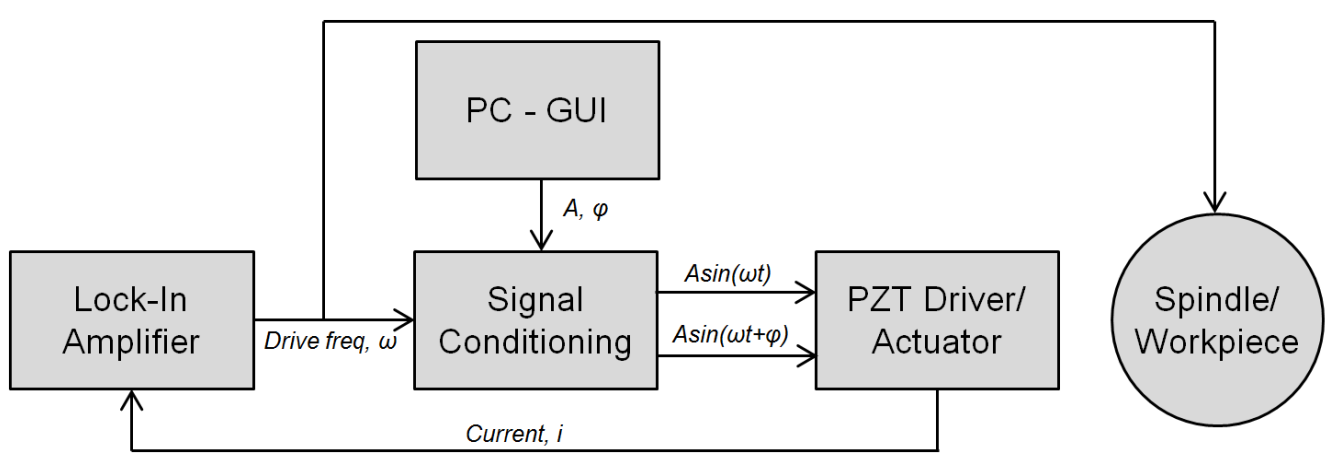

Figure 15. Block diagram of resonant tracking controller where $\omega$ is actuator frequency, $A$ is the voltage amplitude, $\varphi$ is the phase between the two drive signals and $i$ is the current signal measured across the piezo elements.

Since the frequency of the actuator is potentially varying with time, the spindle speed must be synchronized with the drive frequency to maintain the even distribution of indents and minimize feature distortion which occurs when there are large mismatches between the workpiece surface velocity and horizontal indent path velocity. As shown in Figure 15, the drive frequency $\omega$ is fed to the spindle speed controller to modulate the workpiece velocity to compensate for resonant frequency changes. This technique is critical for indenting over large amounts of time (hours), where large changes in resonant frequency can occur (100's of Hz). In practice, the implementation of this synchronization was not necessary due to small workpiece sizes, small indent times (minutes) and only slight resonant frequency changes $(<5 \mathrm{~Hz})$. 


\subsection{Surface error following}

Compensation for roundness of the drum and radial spindle error is performed using an auxiliary piezo actuator that moves a stage containing the indenting actuator based on a cap gauge measurement. Radial error of the drum along the z-axis is measured ahead of the indenting process (at an offset $\mathrm{d}$ of $\sim 0.5 \mathrm{inch}$ ) and the synchronous motion of the mold surface is compensated based on this data as shown in Figure 16. The surface must be tracked to within 50 $\mathrm{nm}$ to maintain uniform indentation coverage.

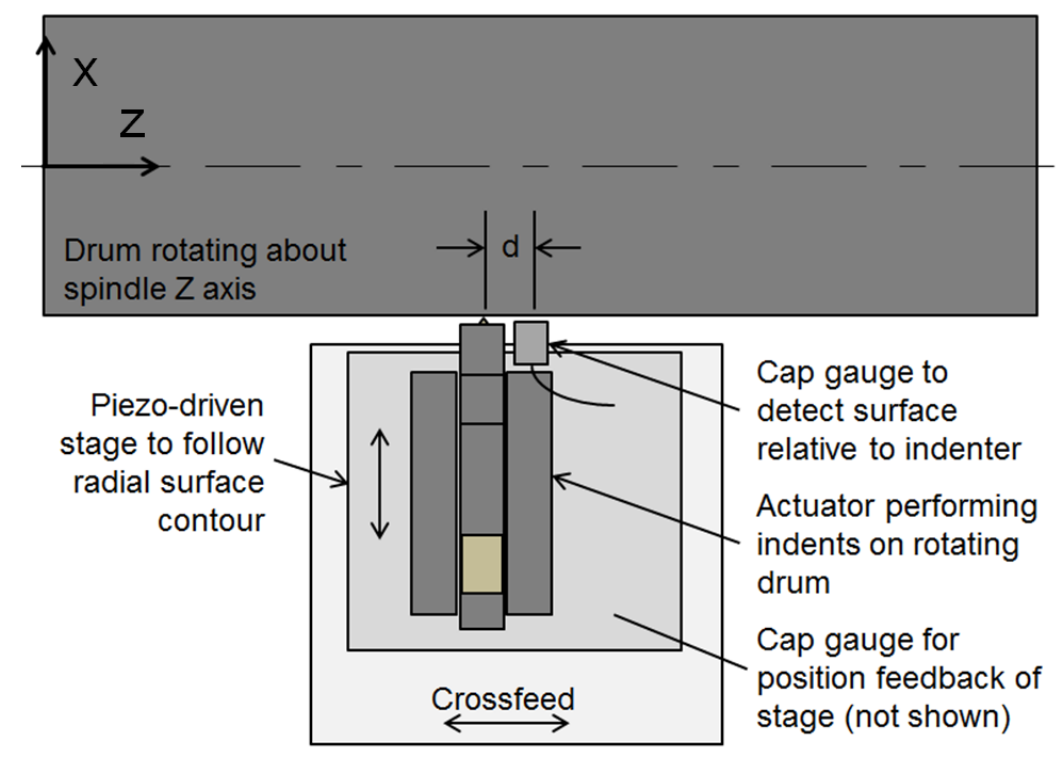

Figure 16. System for tracking radial part error while indenting.

By measuring the surface relative to the actuator, a constant indent depth can be achieved. The position of the actuator could be adjusted using the x-axis of the DTM; however, the closed-loop bandwidth is limited to about $10 \mathrm{~Hz}$. To achieve a higher bandwidth than the DTM axis can achieve, a linear flexure stage was developed to correct the runout and lack of straightness of the workpiece surface. A $50 \mu \mathrm{m}$ range piezoelectric stack is used to make 
position corrections based on an external cap gauge measuring the error in the radial direction. A second cap gauge measures the motion of the flexure stage and forms a closed loop positioning system to track the workpiece measurement based on the external cap gauge. Since the external cap gauge is distance $d$ in the $\mathrm{z}$-direction ahead of the indenting point, the corrective stage actuation must be delayed based on the crossfeed speed. This ensures that the measured error at the cap gauge is being compensated along the z-axis where the indenting is actually being performed.

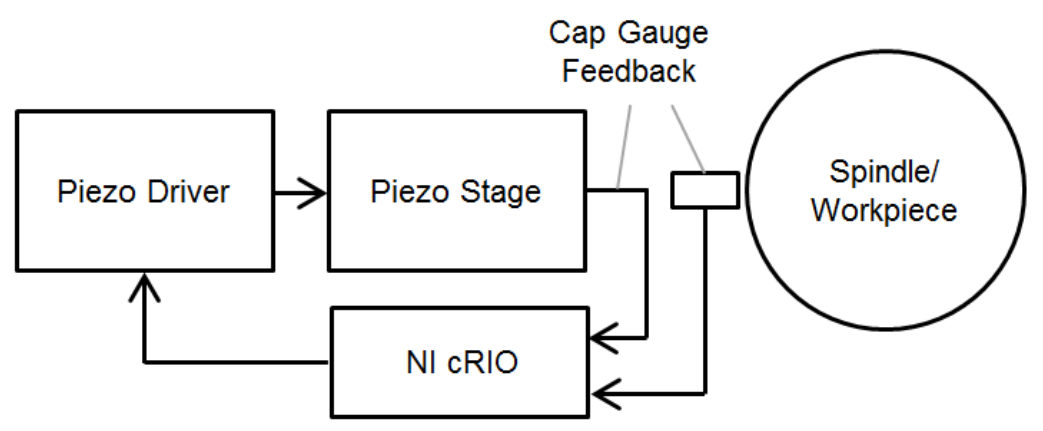

Figure 17. Surface error following schematic for feedback.

A National Instruments compact RIO (cRIO) platform was used to implement a PID controller via FPGA to position the stage based on the surface measurement (Figure ). The bandwidth of the system is filter-limited to $100 \mathrm{~Hz}$ and the rise time for a $1 \mu \mathrm{m}$ step is $0.5 \mathrm{~ms}$. The natural frequency is $2500 \mathrm{~Hz}$. The system was demonstrated using a 1" diameter diamondturned aluminum cylinder with a $1 \mu \mathrm{m}$ arbitrary runout error. Figure 18 shows the part measurement and the corresponding stage cap gauge measurements taken in closed-loop operation, as well as the difference between the measurements that represents tracking error. While rotating at $500 \mathrm{rpm}$, once-per-rev errors are compensated to keep the actuator within 50 nm of the surface. 

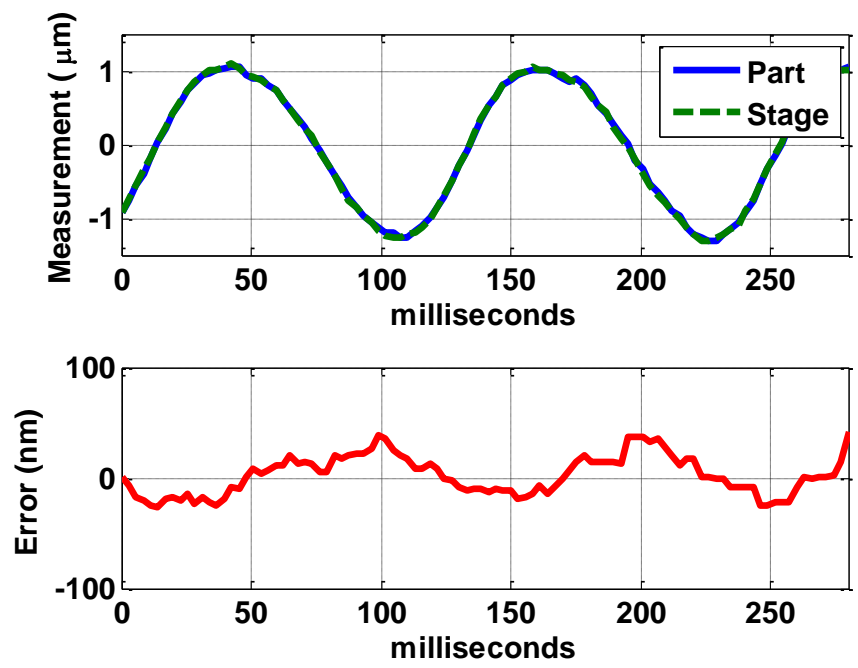

Figure 18. Measured following error of 1 inch diameter cylindrical workpiece.

\section{$5 \quad$ Indentation Experiments}

\subsection{Experimental Setup}

The cylindrical workpiece was mounted on the spindle of a 4-axis DTM (Figure 9(a)) and cut with a single point diamond tool to produce an optical quality finish $(\mathrm{Ra}=10 \mathrm{~nm})$. The actuator is mounted on the $y$-axis and aligned such that the die is square with the periphery of the workpiece. While the spindle is rotating the workpiece, the vibrating actuator is brought into contact with the workpiece using the x-axis, and crossfed in the z-direction to imprint the die pattern along the length of the workpiece. The control and driver electronics from Figure 15 are shown in Figure 19(b), including a Signal Recovery 7270 DSP Lock-in amplifier and two Trek Model PZD M/S piezo drivers. 


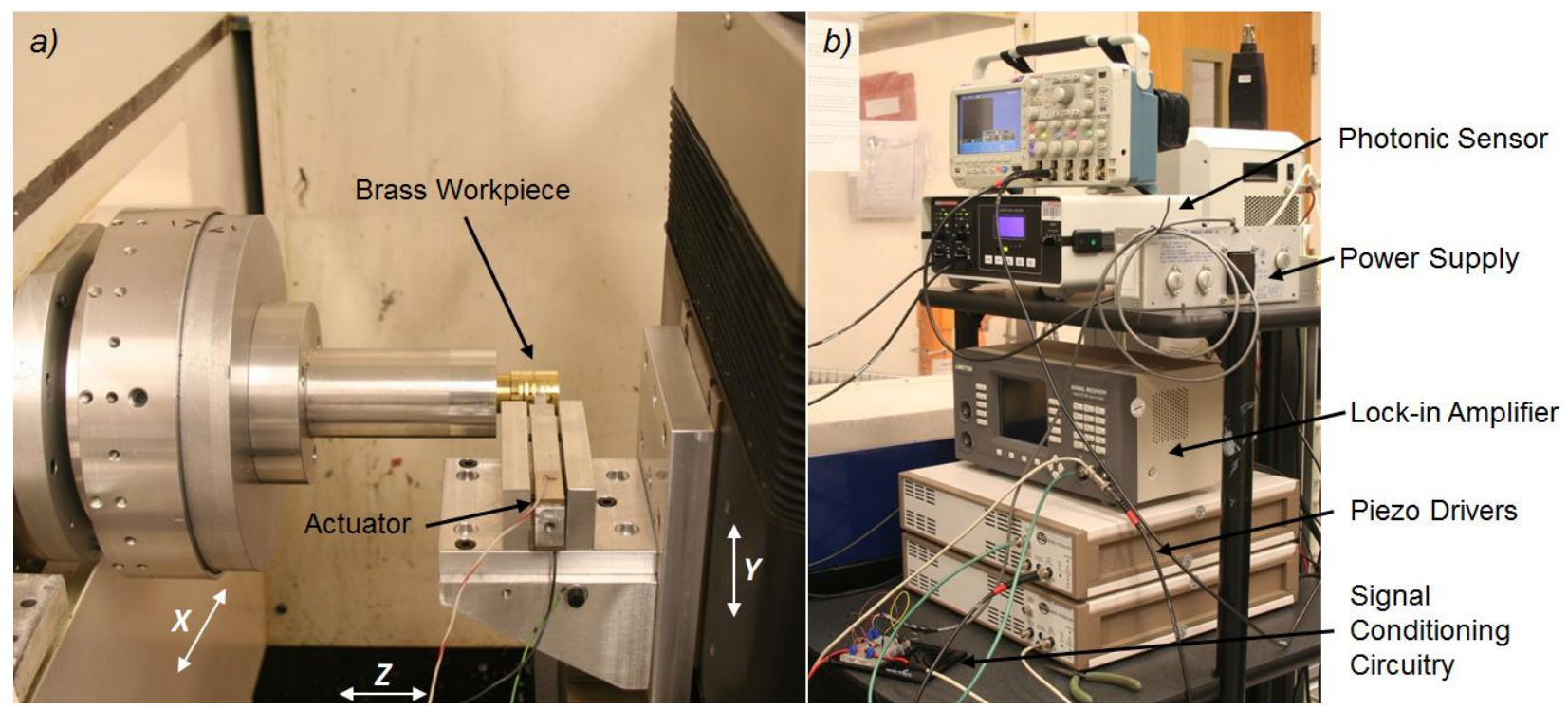

Figure 19. Workpiece and actuator mounted on DTM (a) and electronics to drive and measure actuator (b).

The actuator was driven with a two, $200 \mathrm{~V}$ AC signals that are $100^{\circ}$ degrees out-of-phase to excite both modes simultaneously. The actuator frequency was set to $39,000 \mathrm{~Hz}$, but the actual drive frequency is set by the closed loop control based on the instantaneous current-tovoltage phase measurement (Section 4.1) and is constantly updated to maintain resonance.

To develop the control system, a pair of photonic sensors were used to measure the motion of the actuator normal to the front and top face of the actuator body close to the diamond indenter. A two channel MTI-2100 photonic sensor and a Techtronix MSO2014B oscilloscope are used to monitor the actuator motion in real-time. The elliptical motion of the end of the actuator was optimized using the magnitude and phase of the excitation voltage. The final shape of the die motion used to make the indents is shown in Figure 20. 


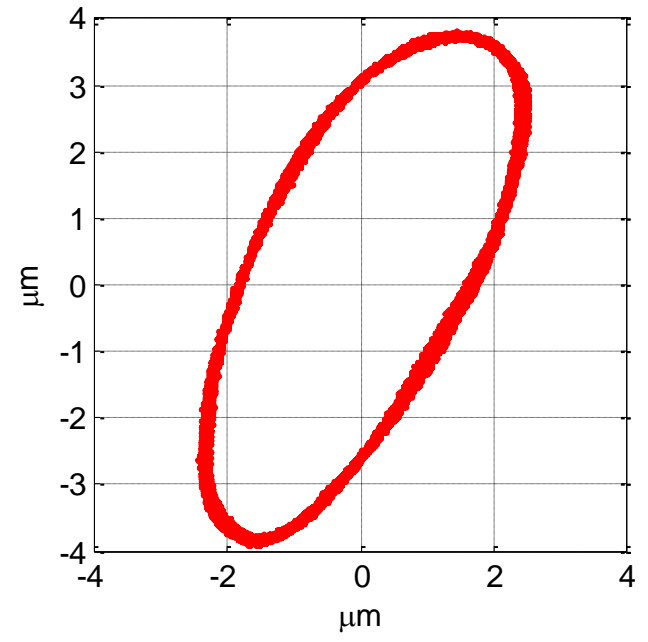

Figure 20. Elliptical indenting motion for a 200V AC input signal

\subsection{Indentations}

Indentations were performed on a 1" diameter 360 brass cylinder at approximately $39 \mathrm{kHz}$ using a $500 \mathrm{~nm}$ pitch die with square features that were $500 \mathrm{~nm}$ tall. The surface speed required to match the transverse motion of the indenting path was $0.297 \mathrm{~m} / \mathrm{s}$. This yields a machined area rate of $5.942 \mathrm{~mm}^{2} / \mathrm{s}$, or approximately 23.7 million $500 \mathrm{~nm}$ pitch features per second. A scanning electron microscope (SEM) is used to image the indented regions of the workpiece. These results are shown in Figure 21. 


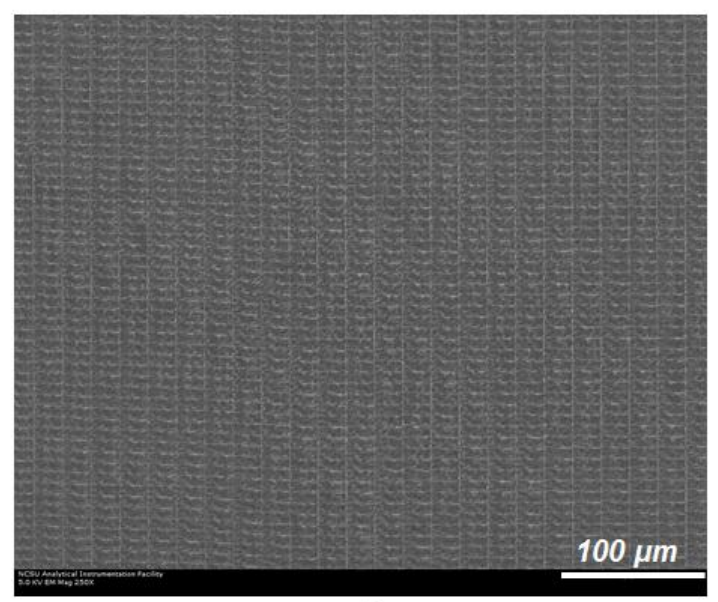

a)

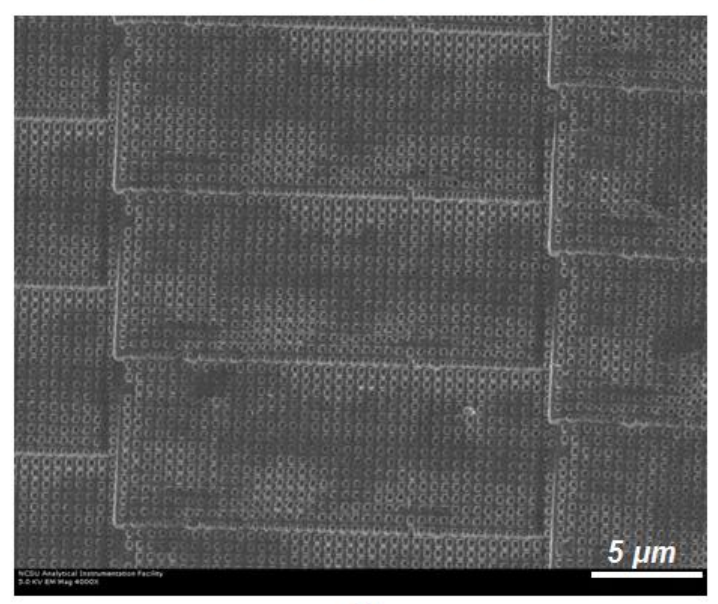

c)

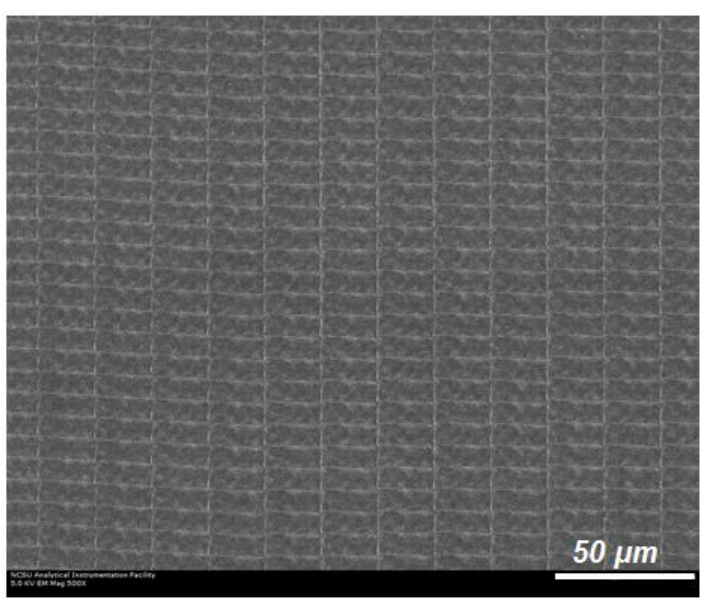

b)

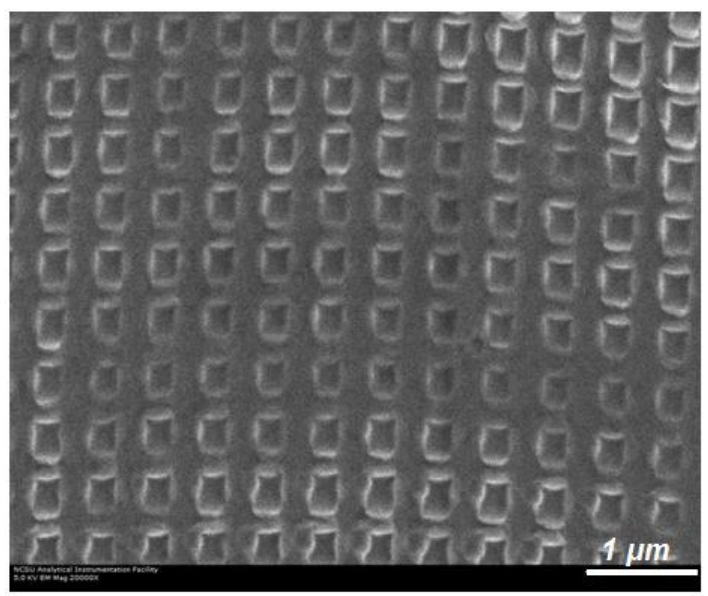

d)

Figure 21. SEM images of indented brass workpiece at 250x (a), 500x (b), 4000x (c), and 20000x (d).

The indents in Figure 21 were generated with the work surface moving from bottom to top, and the actuator feeding from left to right. Each die impression appears rectangular (in spite of using a square die) due to the fact that overlapping was required to match the work surface speed with the current transverse velocity of the actuator. As transverse amplitude of the actuator is increased, larger surface velocities will be used to match the speed.

The lower magnification images in Figure 21(a) and (b) demonstrate the ability to achieve uniform indents on large areas using a resonant actuator and appropriate feedback controller. The lighter and darker regions of the indents are due to material pick-up. Material 
gets pressed into the die and gets caught on the features causing a slight distortion of the surface.

Previous work by Zdanowicz, et al. [10] shows a correlation between material pick-up, feature shape, and work surface hardness. The higher magnification images in Figure (c) and (d) clearly show the impressions left by the square-post die. The indents appear well-formed with minimal distortion, suggesting that the work surface speed matched the velocity of the die at the time of contact. The slight curvature at the 20,000x magnification is caused by charging on the diamond indenter.

The square borders around each indent are artifacts of the die, where a slight tilt in the orientation of the die relative to the workpiece causes one side of the die to indent slightly deeper. The appearance of these border marks could be reduced by reducing the depth of the indents or improving the alignment of the die relative to the surface in subsequent experiments. Since the features appear well-formed, this machined workpiece would act as a suitable mold for replicating these features into a flexible plastic material. Previous work has demonstrated highfidelity feature replication of indented regions using a UV curable plastic [10].

\section{Conclusions}

A $40 \mathrm{kHz}$ piezo-driven, resonant actuator was designed to achieve the desired elliptical vibration path to be used in the high-speed nanocoining process. The performance requirements were outlined and a design methodology proposed based on classical vibrating beam solutions. Finite element analysis was used to shape the actuator such that the longitudinal and transverse vibration modes occur at the same frequency. To combat model errors, a systematic method of tuning the resonant frequencies is proposed and supported with simulations and experimental measurements. Due to resonant frequency drift, a closed loop controller was built to 
automatically track resonance based on the electrical impedance across the piezo elements and maintain a constant elliptical path for generating uniform fields of indents. For runout errors, a system to track the surface of the workpiece and maintain a constant depth of indenting was developed. Indent experiments were performed at $39 \mathrm{kHz}$ on a cylindrical brass workpiece and SEM measurements were used to demonstrate that relatively large areas of well-formed indents are achievable.

\section{$7 \quad$ Acknowledgements}

The authors acknowledge the use of the SEM at NC State's Analytical Instrumentation Facility (AIF) which is supported by the State of North Carolina and the National Science Foundation.

Primary funding for this research was provided by National Science Foundation grant CMMI1000055 monitored by Bruce Krammer. 


\section{References}

[1] Xie G, Zhang G, Lin F, Zhang J, Liu Z, Mu S. The fabrication of subwavelength antireflective nanostructures using a bio-template. Nanotechnology 19(9), pp. 095605. 2008.

[2] Yoshida A, Motoyama M, Kosaku A, Miyamoto K. Antireflective nanoprotuberance array in the transparent wing of a hawkmoth, cephonodes hylas. Zool. Sci. 14(5), pp. 737-741. 1997.

[3] Forberich K, Dennler G, Scharber MC, Hingerl K, Fromherz T, Brabec CJ. Performance improvement of organic solar cells with moth eye anti-reflection coating. Thin Solid Films 516(20), pp. 7167-7170. 2008.

[4] Parker AR, Townley HE. Biomimetics of photonic nanostructures. Nature Nanotechnology 2(6), pp. 347-353. 2007.

[5] Huang J, Wang X, Lin Z. Bio-inspired fabrication of antireflection nanostructures by replicating fly eyes. Nanotechnology 19(2), pp. 025602. 2008.

[6] Gombert A, Glaubitt W, Rose K, Dreibholz J, Bläsi B, Heinzel A, Sporn D, Döll W, Wittwer V. Subwavelength-structured antireflective surfaces on glass. Thin Solid Films 351(1-2), pp. 7378. 1999.

[7] Chen Q, Hubbard G, Shields PA, Liu C, Allsopp E, Wang WN, Abbott S. Broadband motheye antireflection coatings fabricated by low-cost nanoimprinting. Appl. Phys. Lett. 94(26), 2009.

[8] Sun C, Jiang P, Jiang B. Broadband moth-eye antireflection coatings on silicon. Appl. Phys. Lett. 92(6), pp. 061112. 2008.

[9] Park K, Choi HJ, Chang C, Cohen RE, McKinley GH, Barbastathis G. Nanotextured silica surfaces with robust superhydrophobicity and omnidirectional broadband supertransmissivity. ACS Nano 6(5), pp. 3789-3799. 2012.

[10] Zdanowicz E, Dow T, Scattergood R. Rapid fabrication of nanostructured surfaces using nanocoining. Nanotechnology, vol. 23, 2012.

[11] Sherrit S, Dolgin BP, Bar-Cohen Y, Pal D, Kroh J, Peterson T. Modeling of horns for sonic/ultrasonic applications. Presented at Ultrasonics Symposium, IEEE. 1999.

[12] Sherrit S, Askins SA, Gradziol M, Dolgin BP, Bao X, Chang Z, Bar-Cohen Y. Novel horn designs for ultrasonic/sonic cleaning welding, soldering, cutting and drilling. Presented at Proceedings of SPIE. 2002.

[13] Roa SS, Mechanicals Vibrations. New Jersey: Pearson Prentice Hall, 2004 ch. 8, pp. 597614. 
[14] Pons JL, Ochoa P, Villegas M, Fernández JF, Rocon E, Moreno J. Self-tuned driving of piezoelectric actuators: The case of ultrasonic motors. Journal of the European Ceramic Society 27(13-15), pp. 4163-4167. 2007.

[15] Mortimer B, du Bruyn T, Davies J, Tapson J. High power resonant tracking amplifier using admittance locking. Ultrasonics 39(4), pp. 257-261. 2001.

[16] Fiorillo AS. Piezo-polymer ultrasound transducer with controlled resonance for medicine and robotics. Proc. IEEE Ultrasonics Symp. pp. 729-734. 1998.

[17] Abdullah A, Pak A, Shahidi A. "Equivalent Electrical Simulation of High-Power Ultrasonic Piezoelectric Transducers by Using Finite Element Analysis."

[18] Maruyama Y, Takasaki M, Mizuno T. "Resonance frequency tracing system for langevin type ultrasonic transducers," in Mechatronic Systems Simulation Modeling and Control, Eds. InTech, 2010.

[19] Mizutani Y, Suzuki T, Hiroaki I, Yoshida H. "Power Maximizing of Ultrasonic Transducer Driven by MOS-FET Inverter Operating at $1 \mathrm{MHz}$ " vol. 6, 1996.

[20] Li Q, Zhu L, Wang F. Design of ultrasonic generator based on DDS and PLL technology. Presented at High Density Packaging and Microsystem Integration, 2007. HDP '07. International Symposium On. 2007.

[21] Shamoto E, Suzuki N, Naoi Y, Development of untrasonic elliptical vibration controller for elliptical vibration cutting, CIRP Ann, 2002; 51:327-330.

[22] Shamoto E, Moriwaki T, Study on Elliptical Vibariont Cutting, Annals of the CIRP, Vol 43, pg 35-38, 1994.

[23] Brehl, D and Dow, TA, Review of vibration-assisted machining, Precision Engineering, 32 (2008) 153-172

[24] Bauer, M, Design of a Linear High Precision Ultrasonic Piezoelectric Motor, PhD Dissertation, NC State University, 2001. 